\title{
OTOLOGY
}

\section{Multi-option therapy vs observation for small acoustic neuroma: hearing-focused management}

\author{
Opzioni di terapia vs osservazione per i piccoli neurinomi dell'acustico: \\ una gestione orientata alla funzione uditiva
}

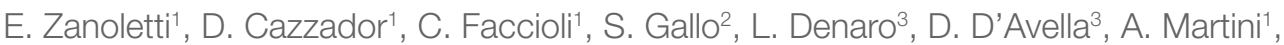 \\ A. Mazzoni ${ }^{1}$ \\ Department of Neurosciences, Otorhinolaryngology Unit, ${ }^{2}$ Department of Neurosciences, Audiology Unit, \\ ${ }^{3}$ Department of Neurosciences, Unit of Neurosurgery, University of Padua, Italy
}

\section{SUMMARY}

The current treatment options for acoustic neuromas (AN) - observation, microsurgery and radiotherapy - should assure no additional morbidity on cranial nerves VII and VIII. Outcomes in terms of disease control and facial function are similar, while the main difference lies in hearing. From 2012 to 2016, 91 of 169 patients (54\%) met inclusion criteria for the present study, being diagnosed with unilateral, sporadic, intrameatal or extrameatal AN up to $1 \mathrm{~cm}$ in the cerebello-pontine angle; the remaining 78 patients (46\%) had larger AN and were all addressed to surgery. The treatment protocol for small AN included observation, translabyrinthine surgery, hearing preservation surgery (HPS) and radiotherapy. Hearing function was assessed according to the Tokyo classification and the American Academy of Otolaryngology-Head and Neck Surgery (AAO-HNS) classification. Sixty-one patients (71\%) underwent observation, 19 (22\%) HPS and 6 (7\%) translabyrinthine surgery; 5 patients were lost to follow-up. Median follow-up was 25 months. In the observation group, $24.6 \%$ of patients abandoned the wait-and-see policy for an active treatment; the risk of switching from observation to active treatment was significant for tumour growth $(\mathrm{p}=0.0035)$ at multivariate analysis. Hearing deteriorated in $28 \%$ of cases without correlation with tumour growth; the rate of hearing preservation for classes C-D was higher than for classes A-B $(\mathrm{p}=0.032)$. Patients submitted to HPS maintained an overall preoperative hearing class of Tokyo and AAO-HNS in $63 \%$ and $68 \%$ of cases, respectively. Hearing preservation rate was significantly higher for patients presenting with preoperative favourable conditions (in-protocol) $(\mathrm{p}=0.046)$. A multi-option management for small AN appeared to be an effective strategy in terms of hearing outcomes.

KEY WORDS: Acoustic neuroma $\bullet$ Vestibular schwannoma $\bullet$ Wait and see $\bullet$ Hearing preservation $\bullet$ Microsurgery

\section{RIASSUNTO}

Le opzioni di trattamento per il neurinoma del nervo acustico (AN), ovvero osservazione, microchirurgia e radioterapia non dovrebbero esporre a una morbidità addizionale i nervi cranici VII e VIII. Esse comportano risultati simili su controllo del tumore e conservazione del nervo facciale, ma differenti e discussi quanto a udito. Dal 2012 al 2016, 169 pazienti con diagnosi di AN sporadico, monolaterale sono stati valutati nel nostro centro. In totale, 91 pazienti hanno soddisfatto i criteri di inclusione, presentando un tumore intracanalare o extrameatale di dimensioni inferiori a $1 \mathrm{~cm}$ in angolo pontocerebellare. I restanti 78 pazienti con AN di dimensioni maggiori sono stati tutti trattati con opzione chirurgica primaria. La funzione uditiva è stata determinata sulla base delle classificazioni di Tokyo e dell'American Academy of Otolaryngology-Head and Neck Surgery (AAO-HNS). Il controllo post trattamento ha avuto una durata media di 25 mesi. Sessantuno pazienti (71\%) sono andati incontro a trattamento conservativo di osservazione, $19(22 \%)$ a chirurgia di preservazione dell'udito (HPS) e 6(7\%) a chirurgia per via translabirintica; 5 pazienti sono stati esclusi per incompletezza di dati. Il 24,6\% dei pazienti in osservazione è uscito da tale protocollo durante il follow-up. Il rischio di cambiare da osservazione a trattamento attivo è risultato significativo all'analisi multivariata per i tumori in crescita $(p=0,0035)$. Nello stesso gruppo in osservazione, l'udito è andato incontro a un peggioramento nel $28 \%$ dei casi, indipendentemente da una correlazione con la crescita tumorale. La conservazione di un udito in classe A-B si è dimostrata significativamente inferiore rispetto a quella per un udito in classe C-D $(p=0,032)$ nel tempo di osservazione. I pazienti sottoposti a HPS hanno mantenuto la classe uditiva preoperatoria nel 63\% e nel 68\% dei casi, rispettivamente per le classificazioni di Tokyo e AAO-HNS. Il tasso di preservazione dell'udito inoltre, si è dimostrato significativamente maggiore per i pazienti che si presentavano con caratteristiche preoperatorie più favorevoli (in-protocol) $(p=0,046)$. Un protocollo di trattamento multi-opzionale per $i$ piccoli AN rappresenta una strategia efficace per il risultato chirurgico a breve termine.

PAROLE CHIAVE: Neurinoma dell'acustico $\bullet$ Schwannoma vestibolare $\bullet$ Wait and scan $\bullet$ Preservazione dell'udito $\bullet$ Microchirurgia 


\section{Introduction}

Thanks to early imaging, acoustic neuromas (AN) are now diagnosed more frequently and are often small, presenting with mild symptoms. The natural history of the tumour supports abstaining from any treatment, shifting to an active policy if it grows or new, invalidating symptoms develop ${ }^{12}$. Active therapies such as microsurgery or radiotherapy (RT) may be offered at the time of diagnosis, but only if they provide the patient more benefit than observation alone ${ }^{34}$. It is essential to ask why and when a small AN should be actively treated. The goal of treatment should be to assure long-term cure of the disease with no further morbidity on cranial nerves VII and VIII, or post-treatment sequelae.

The three possible treatment options to consider - observation, microsurgery and $\mathrm{RT}^{3}-$ are not equivalent. The value of each is influenced by the goals of treatment, the patient's expectations and the institution's treating attitude. Previous reports showed similar outcomes in terms of tumour removal and facial function, while the main difference lies in hearing function ${ }^{3-5}$. All three treatment approaches will involve a substantial loss of serviceable hearing over time ${ }^{3-5}$. Surgery has various rates of early hearing loss, while observation and RT reportedly have longer-term serviceable hearing rates ${ }^{3}$. Active treatments may be worthwhile if they can preserve hearing better than long-term observation. Hearing preservation surgery (HPS) is warranted as soon as growth is evident ${ }^{4}$.

At our institution, the treatment options currently consist in observation alone, HPS, traditional surgery (possibly associated with hearing rehabilitation with cochlear implants [CI]), and RT. These four options have been adopted in our protocol ${ }^{6}$ (Table I) with a view to offering the best chance of long-term cure and preservation of facial function and hearing.

The aim of the present study was to analyse and provide an extended view of the treatment strategies that are available when a small AN is diagnosed, focusing on hearing as the at-risk function in the different therapies. A multi- optional treatment was offered to each patient, the choice being based on pre-defined parameters, as well as patient willingness.

\section{Materials and methods}

\section{Participants}

From January 2012 to June 2016, 169 patients with a first diagnosis of unilateral, sporadic AN were assessed at our institution. Patients diagnosed with neurofibromatosis type 2 or cystic AN were not included. A prospectively-maintained clinical database and the Italian National Health System's electronic medical records were queried for all patients. Only small AN were considered in the present study, i.e. intrameatal or extrameatal tumours up to $1 \mathrm{~cm}$ in size in the cerebello-pontine angle (CPA). A total of 91 of 169 patients met this inclusion criteria.

\section{Diagnosis}

AN were diagnosed on high-resolution contrast-enhanced magnetic resonance imaging (MRI). Tumour size was measured in the longest diameter $(\mathrm{mm})$ in the CPA on contrastenhanced $\mathrm{T} 1$ sequences ${ }^{7}$. Hearing was assessed with pure tone audiometry, speech audiometry and auditory brainstem responses (ABR). The hearing measures considered were pure tone average (PTA) from 500 to $4000 \mathrm{~Hz}$ and speech discrimination score (SDS) obtained at an intensity of $40 \mathrm{~dB}$ above detection or at most comfortable threshold. The results were stratified according to the Tokyo classification ${ }^{7}$. Hearing outcome is given with the American Academy of Otolaryngology-Head and Neck Surgery (AAO-HNS) classification ${ }^{8}$, where necessary for the purpose of comparisons with data in the literature. Facial nerve function was assessed clinically according to the House-Brackmann (HB) grading system ${ }^{9}$ and using electromyography.

\section{Treatment options}

The treatment options for small AN (Table I) included observation, RT, HPS and translabyrinthine surgery. Indi-

Table I. Institutional protocol for small sporadic acoustic neuroma management (from Martini et al., $2017^{6}$, mod.).

\begin{tabular}{lll}
$\begin{array}{l}\text { Acoustic neuroma size } \\
(\mathbf{m m} \text { in the CPA angle) }\end{array}$ & Decision factors & Treatment \\
$<10 \mathrm{~mm}$ & Good hearing & Hearing preservation surgery \\
& $(<30 \mathrm{~dB},>70 \% \mathrm{SDS}$, normal or slightly modified ABR) & or observation* \\
& Good hearing & Observation \\
& $(<30 \mathrm{~dB},>70 \% \mathrm{SDS}$, normal or slightly modified ABR) & \\
& + surgical risk/unwillingness to undergo surgery & \\
& Poor hearing & Observation* or surgery and hearing rehabilitation with \\
& $(>30 \mathrm{~dB},<70 \% \mathrm{SDS})$ & cochlear implant \\
\hline
\end{tabular}

${ }^{*}$ Active treatment (surgery or RT) in the event of tumour growth to $>15 \mathrm{~mm}$, or vertigo, or VII cranial nerve impairment. CPA: cerebello-pontine angle 
vidual cases were first discussed, balancing the pros and cons of each option, and the final choice proposed to the patient was based on: (1) tumour size; (2) patient's age and aging (comorbidities); (3) hearing status; (4) tumour growth (defined as stable, growing, or not assessed); (5) contralateral hearing function; (6) facial nerve function; (7) patient's willingness, and other personal aspects such as work, social life and follow-up availability.

Hearing rehabilitation with CI was discussed at the time of planning the therapy, and was considered both in the event of HPS failure and in cases of early translabyrinthine surgery, when the cochlear nerve was preserved. The outcome of patients treated with any type of hearing rehabilitation goes beyond the scope of the present study.

\section{Wait-and-see patients}

Observation was indicated in principle for tumours coinciding with hearing impairment (class $\mathrm{C}$ or worse on the Tokyo scale), for aged/aging patients, or for patients with good hearing function but unwilling/unable to undergo surgery. Patients under observation underwent clinical examination, pure tone- and speech audiometry and contrast-enhanced MRI planned at 6-month intervals for the first year, then yearly for the next 5 years. The followup was then planned every 3 years afterwards. Tumour growth was defined as an increase of more than $2 \mathrm{~mm}$ in the tumour's largest extrameatal diameter between the first and latest MRI scans. Hearing deterioration was defined as hearing class change/worsening at the last evaluation. Regarding HPS, it was applied only to cases with good hearing (class AB Tokyo) at diagnosis.

\section{Hearing preservation surgery (HPS)}

The indications for HPS included class A-B hearing on the Tokyo scale, normal ABR or slightly increased waves III and V (10) and intrameatal or $\leq 10 \mathrm{~mm}$ tumours in the CPA, regardless of their growth and extension to the fundus. Patients who met these inclusion criteria were assigned to an in-protocol group for HPS. Patients strongly motivated to receive HPS, but not satisfying one or more of the above inclusion criteria, were assigned to an offprotocol group. A retro-sigmoid approach with a retrolabyrinthine meatotomy was adopted in all cases of HPS ${ }^{11} 12$.

\section{Translabyrinthine surgery}

Translabyrinthine surgery was only considered as a primary treatment option in the case of invalidating vestibular symptoms or facial nerve weakness at the time of diagnosis. Otherwise, translabyrinthine surgery was discussed as a secondary option during the observation period in the event of: 1 . the onset of invalidating vertigo, or facial nerve weakness; 2 . tumour growth with impaired hearing (class $\mathrm{C}$ or worse on the Tokyo scale); 3. impaired hearing in cases where functional hearing rehabilitation surgery with CI was planned. The follow-up for surgically-treated patients involved contrast-enhanced MRI scheduled 1 and 3 years after surgery. Further imaging was then planned 6 , 10 and 15 years afterwards.

\section{Radiotherapy}

No tumour was submitted to primary RT unless there was evidence of growth ${ }^{6}$. Patients with good hearing at diagnosis never received RT as first-choice primary treatment.

\section{Statistical analysis}

Categorical variables are presented as frequencies and percentages, continuous data as means and standard deviations or as medians and interquartile range (IQR), according to the Shapiro-Wilk test of normality. Pearson's chi-square test or Fisher's exact test were used to compare categorical variables. The Mann-Whitney $U$ test or the t-test for independent and paired samples were applied to continuous variables, depending on their distribution. The cumulative hazard of hearing impairment was calculated in the wait-and-see group using the Kaplan-Meier method. In this observation group, the association of 5 variables (age, gender, tumour site, tumour growth, and hearing impairment) with the risk of the conservative

Table II. Characteristics of the study population, stratified by tumour site at diagnosis.

\begin{tabular}{|c|c|c|c|}
\hline & $\begin{array}{c}\mathrm{IAC}(\mathrm{n}=47) \\
\mathrm{n}(\%)\end{array}$ & $\begin{array}{c}<10 \mathrm{~mm} \mathrm{CPA}(\mathrm{n}=39) \\
\mathrm{n}(\%)\end{array}$ & $p$ value \\
\hline Male & $28(59.5)$ & $20(51)$ & 0.441 \\
\hline Age (years), mean \pm SD & $56.2 \pm 12.6$ & $55.2 \pm 12.7$ & 0.697 \\
\hline Baseline PTA $(\mathrm{dB})$, mean $\pm \mathrm{SD}$ & $43.2 \pm 21.2$ & $41.4 \pm 22.7$ & 0.704 \\
\hline Tokyo class A-B hearing & $24(51)$ & $19(49)$ & 0.829 \\
\hline Observation & $34(72)$ & $27(69)$ & 0.752 \\
\hline HPS & $9(19)$ & $10(26)$ & 0.470 \\
\hline Translabyrinthine surgery & $4(8.5)$ & $2(5)$ & 0.685 \\
\hline
\end{tabular}

IAC: internal auditory canal; CPA: cerebello-pontine angle; PTA: pure tone average; HPS: hearing preservation surgery. 
treatment being abandoned was tested with the log-rank test and Cox regression. Statistical significance was assumed when $\mathrm{p}<0.05$ in two-tailed tests, hazard ratios (HR) and confidence intervals at 95\% (95\% CI) are reported. The statistical analysis was performed using the Statistical Package for the Social Sciences software (IBM SPSS Statistics for Windows, version 20, IBM Corp., Armonk, N.Y., USA).

\section{Results}

Of 169 patients presenting with sporadic AN, 91 met our inclusion criteria. Five had incomplete medical records and were consequently excluded, leaving 86 patients for the final analysis.

\section{Patient demographics, signs and symptoms}

The sample included 38 female $(44.2 \%)$ and 48 male $(55.8 \%)$ with a mean age at diagnosis of $55.7 \pm 12.6$ years. At diagnosis, more than one symptom was reported by $38.5 \%$ of patients. Hearing loss was present in $56 \%$ of cases, followed by tinnitus (37\%), vertigo (29\%) and facial nerve palsy (1\%). The most common combinations were hearing loss and tinnitus (35\%), or vertigo and tinnitus $(27 \%)$. Eighty-five patients $(99 \%)$ presented with a facial nerve function at diagnosis of grade $\mathrm{I} \mathrm{HB}$, and one patient with grade II HB.

The treatment strategies adopted at the time of diagnosis are shown in Figure 1. The findings are analysed and grouped by: (1) tumour size at diagnosis; and (2) treatment strategy adopted at diagnosis (observation, surgery, or RT). The median follow-up was 25 months (IQR 12-38 months).

\section{Tumour size}

Forty-seven patients $(54.6 \%)$ had a pure intrameatal tumour at diagnosis, while 39 patients $(45.4 \%)$ presented with extrameatal tumours (Table II). The two groups did not differ significantly in terms of sex, age or hearing status (PTA and Tokyo scores) at presentation. Moreover, tumour size at diagnosis did not influence the initial treatment policy.

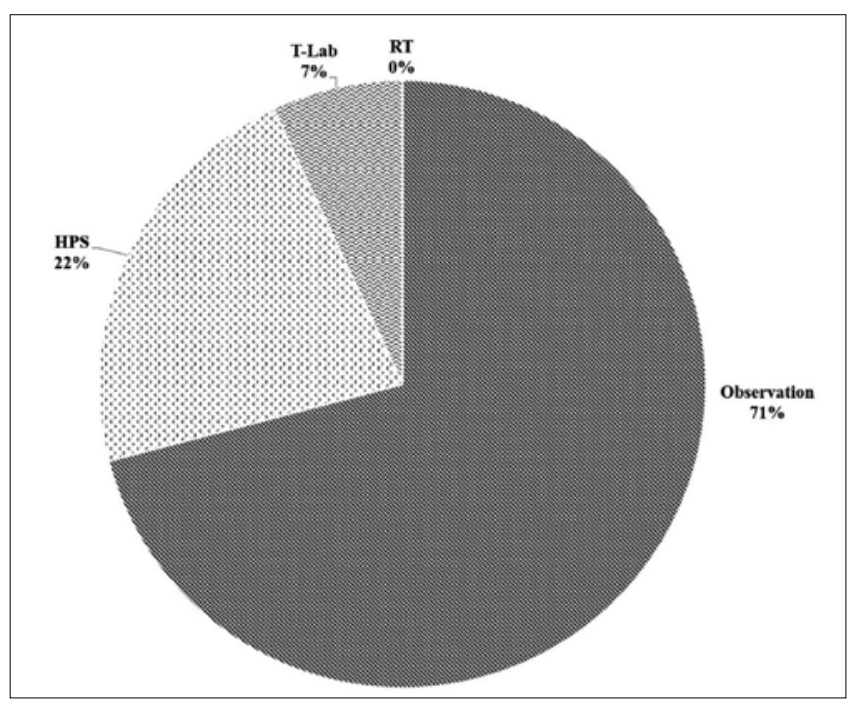

Fig. 1. Distribution of treatment policies adopted for small acoustic neuromas at diagnoses.

\section{Treatment strategies \\ 2.1 Wait-and-see}

The observation group included 61 patients, 30 female $(49 \%)$ and 31 male $(51 \%)$, with a mean age of $58.9 \pm 11.4$ years. There were 34 cases of intrameatal AN (55.7\%), while 27 patients $(44.3 \%)$ had extrameatal tumours (Table III).

At last observation, tumour growth occurred in 17 patients (28\%). Of these, $11(65 \%)$ switched to active treatment, while $6(35 \%)$ remained under observation. As shown in Figure 2, the cumulative hazard of tumour growth after diagnosis was $3.4 \%$ for intrameatal tumours and $15.6 \%$ for extrameatal tumours in the first year, rising to $12.3 \%$ and $26.2 \%$, respectively, in the first two years $(\mathrm{HR}=1.93$, 95\% CI $=0.73-5.04 ; \mathrm{p}=0.173$ ).

Analysing the overall outcome of conservative management, 46 patients $(75.5 \%)$ remained under observation, $12(19.5 \%)$ underwent surgical procedures, and 3 patients (5\%) were addressed to RT. Of 12 patients submitted to

Table III. Patients' characteristics in the wait-and-see group, by tumour site at diagnosis.

\begin{tabular}{|c|c|c|c|}
\hline & $\begin{array}{c}\mathrm{IAC}(\mathrm{n}=34) \\
\mathrm{n}(\%)\end{array}$ & $\begin{array}{c}<10 \mathrm{~mm} \text { CPA }(\mathrm{n}=27) \\
\mathrm{n}(\%)\end{array}$ & $p$ value \\
\hline Male & $18(53)$ & $13(48)$ & 0.710 \\
\hline Age (years), mean \pm SD & $59.1 \pm 11.5$ & $59.5 \pm 10.8$ & 0.908 \\
\hline Baseline PTA $(\mathrm{dB})$, mean $\pm \mathrm{SD}$ & $45.2 \pm 21.1$ & $48.0 \pm 19.2$ & 0.597 \\
\hline Tokyo class A-B hearing & $15(44)$ & $9(33)$ & 0.392 \\
\hline Tumour growth & $7(21)$ & $10(37)$ & 0.155 \\
\hline Hearing deterioration & $11(32)$ & $6(22)$ & 0.381 \\
\hline Facial nerve loss & $0(-)$ & $0(-)$ & - \\
\hline
\end{tabular}

IAC: internal auditory canal; CPA: cerebello-pontine angle; PTA: pure tone average 


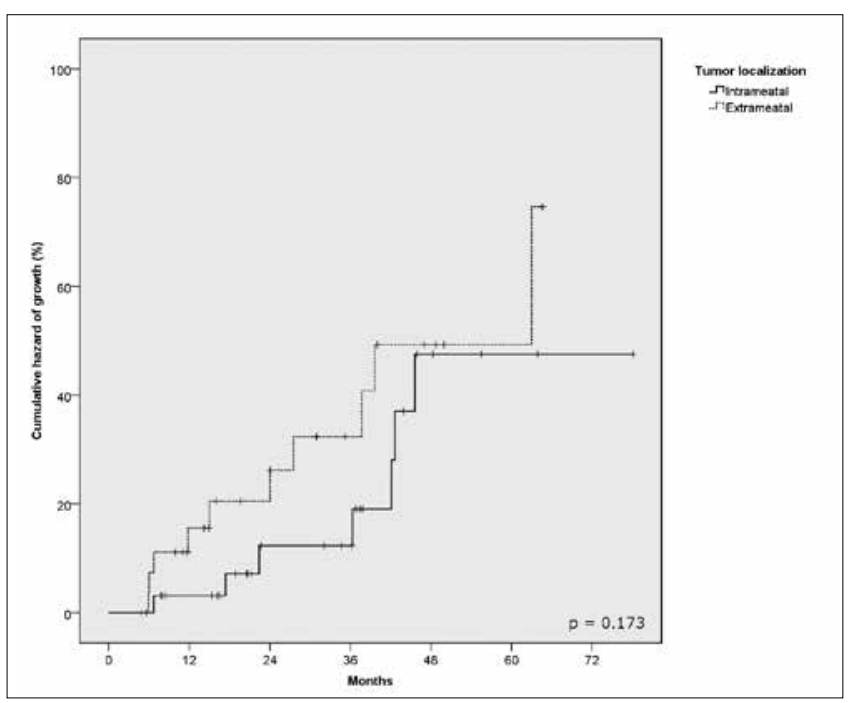

Fig. 2. Cumulative hazard of tumour growth by site at diagnoses.

surgery, all the 11 cases treated with a translabyrinthine approach had extrameatal growth, and the only case with growing intralabyrinthine symptomatic tumour was operated on through a transcanal approach to the vestibule.

The risk of switching from observation to active treatment was significant at univariate analysis (Fig. 3, Table IV) for site at diagnosis $(\mathrm{p}=0.0226)$ and tumour growth $(\mathrm{p}=0.0004)$. Hearing status did not influence the course of the observation treatment policy $(\mathrm{p}=0.873)$. On multivariate analysis (Table IV), only tumour growth was confirmed as having an impact on the probability of remaining under observation, and prompting the switch to active treatment (surgery or RT).

Surgery was indicated for patients initially managed with the wait-and-see policy as a result of: tumour growth (8 cases); patient preference (2 cases); hearing loss (1 case); onset of intractable vertigo (1 case).
Three patients were referred for RT after observation due to tumour growth over a median observation period of 17.4 months (IQR 16-21 months). In two cases, cyberknife treatment was preferred due to the patients' age (mean 70.4 years), comorbidities and impaired hearing status (both class D). The last patient addressed to RT was 56 years old and had a preserved hearing function (class A), but preferred a non-surgical treatment over HPS. After RT, all patients showed disease control in terms of absence of tumour growth at radiological imaging after a mean follow-up of 27.6 months. Hearing function decline was observed in the only patient whose hearing was good at diagnosis.

\subsubsection{Hearing outcome in the wait-and-see group}

At diagnosis, 24 patients (39.4\%) showed preserved hearing, defined as Tokyo class A-B. The other 37 patients were in hearing classes $\mathrm{C}(27.8 \%), \mathrm{D}(16.4 \%), \mathrm{E}(9.8 \%)$, or $\mathrm{F}(6.6 \%)$. There was a significant difference $(\mathrm{p}<0.001)$ between median PTA at diagnosis and latest hearing assessment with $43 \mathrm{~dB}$ (IQR 31-57 dB) and $53 \mathrm{~dB}$, (IQR 40-62.5 dB), respectively.

Hearing deteriorated in 17 patients (28\%). It is noteworthy that hearing deterioration coincided with growing tumours in only 6 cases $(35 \% ; \mathrm{p}=0.52)$. As shown in Figure 4, the cumulative hazard of hearing impairment related to tumour growth was $4.8 \%$ for stable tumours and $12.5 \%$ for growing tumours in the first year, and $11.9 \%$ and $22.2 \%$, respectively, in the first two years $(\mathrm{HR}=1.82$; $95 \% \mathrm{CI}=0.59-5.66 ; \mathrm{p}=0.214)$.

Preoperative classes A and B were maintained in 58\% of cases at last follow-up examination, and preoperative classes $C$ and $D$ in $85 \%$ of cases $(p=0.032)$. Figure 5 shows a statistical trend in hearing deterioration after the first 24 months of observation, which was higher for patients in classes A or B at diagnosis, compared to patients in classes $\mathrm{C}$ and $\mathrm{D}(\mathrm{HR}=2.67 ; 95 \% \mathrm{CI}=0.93-7.63 ; \mathrm{p}=0.081)$.

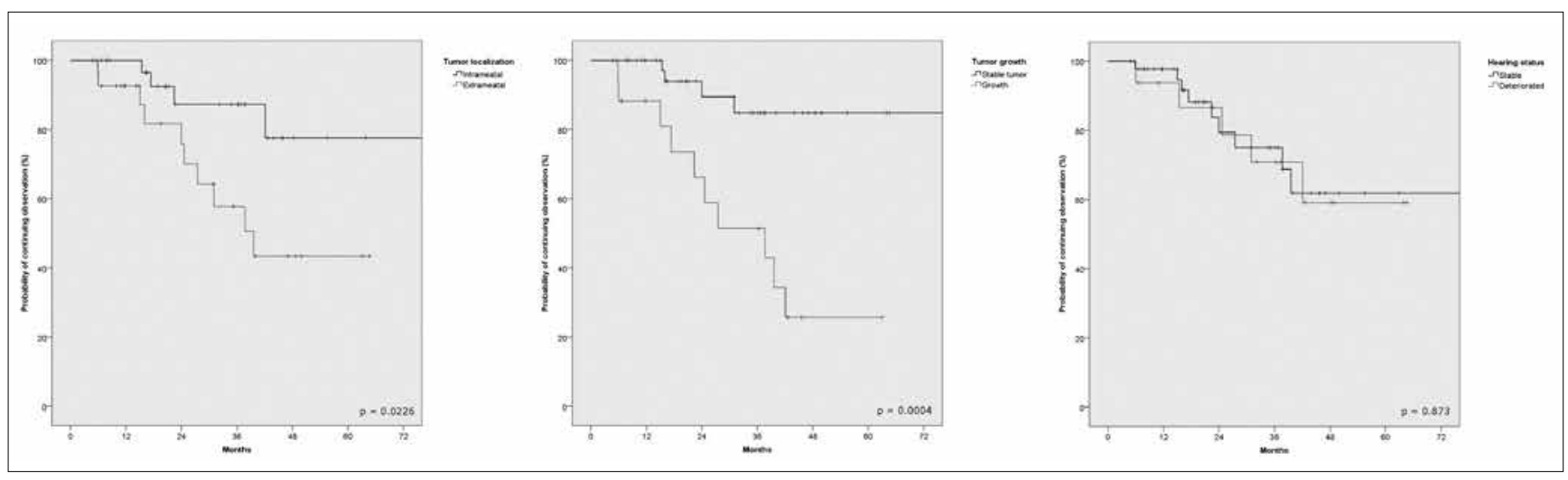

Fig. 3. Probability of remaining under observation by tumour site at diagnoses, tumour growth and hearing deterioration during follow-up. 
Table IV. Univariate and multivariate analysis of predictors for switching from the wait-and-see strategy to active treatment.

\begin{tabular}{|c|c|c|c|}
\hline Variable & HR & $95 \% \mathrm{Cl}$ & $p$ value \\
\hline \multicolumn{4}{|l|}{ Univariate analysis } \\
\hline $\begin{array}{l}\text { Age }(y) \\
<65 \\
\geq 65\end{array}$ & $\begin{array}{l}1.00 \\
0.80\end{array}$ & $\begin{array}{c}R G \\
0.28-2.26\end{array}$ & 0.675 \\
\hline $\begin{array}{l}\text { Sex } \\
\text { Male } \\
\text { Female }\end{array}$ & $\begin{array}{l}1.00 \\
0.94\end{array}$ & $\begin{array}{c}R G \\
0.34-2.65\end{array}$ & 0.912 \\
\hline $\begin{array}{l}\text { Tumour site } \\
\text { Intrameatal } \\
\text { Extrameatal }\end{array}$ & $\begin{array}{l}1.00 \\
3.16\end{array}$ & $\begin{array}{c}R G \\
1.12-8.94\end{array}$ & $0.0226^{\star}$ \\
\hline $\begin{array}{l}\text { Tumour growth } \\
\text { No } \\
\text { Yes }\end{array}$ & $\begin{array}{l}1.00 \\
5.54\end{array}$ & $\begin{array}{c}R G \\
1.74-17.07\end{array}$ & $0.0004^{*}$ \\
\hline $\begin{array}{l}\text { Hearing } \\
\text { impairment } \\
\text { No } \\
\text { Yes }\end{array}$ & $\begin{array}{l}1.00 \\
1.09\end{array}$ & $\begin{array}{c}R G \\
0.36-3.24\end{array}$ & 0.873 \\
\hline \multicolumn{4}{|c|}{ Multivariate analysis } \\
\hline $\begin{array}{l}\text { Tumour site } \\
\text { Intrameatal } \\
\text { Extrameatal }\end{array}$ & $\begin{array}{l}1.00 \\
3.09\end{array}$ & $\begin{array}{c}R G \\
0.96-9.96\end{array}$ & 0.058 \\
\hline $\begin{array}{l}\text { Tumour growth } \\
\text { No } \\
\text { Yes }\end{array}$ & $\begin{array}{l}1.00 \\
5.66\end{array}$ & $\begin{array}{c}R G \\
1.76-18.15\end{array}$ & $0.0035^{\star}$ \\
\hline
\end{tabular}

HR: hazard ratios; Cl: confidence intervals; RG: reference group; *: statistical significance

\subsection{Surgery}

The surgical group included 37 patients, 14 female (37.8\%) and 23 male $(62.2 \%)$, with a mean age of $50 \pm 12.4$ years. There were 17 cases $(46 \%)$ treated with translabyrinthine surgery, $19(51.4 \%)$ with HPS, and 1 (2.6\%) via a trans-

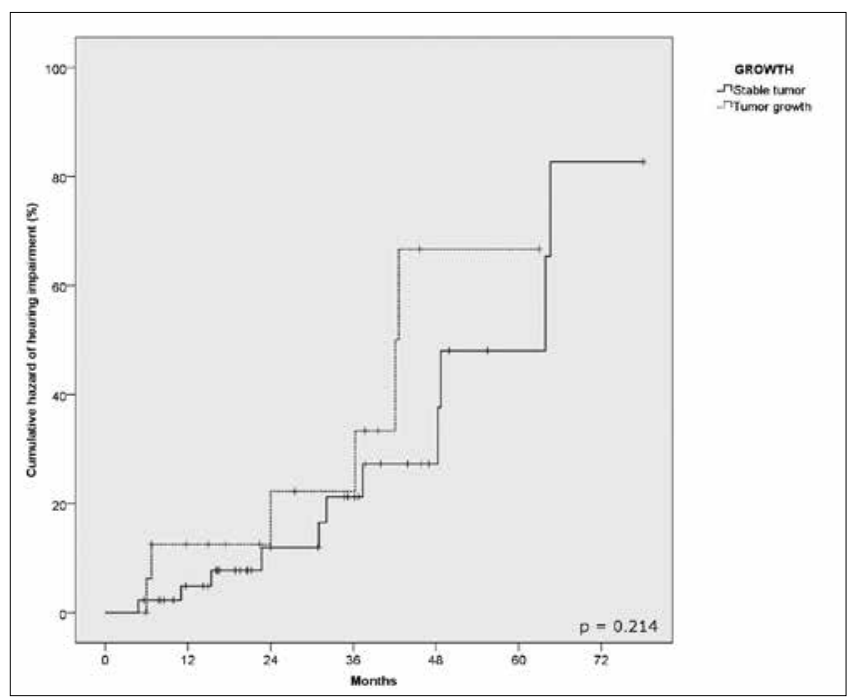

Fig. 4. Cumulative hazard of hearing impairment related to tumour growth.

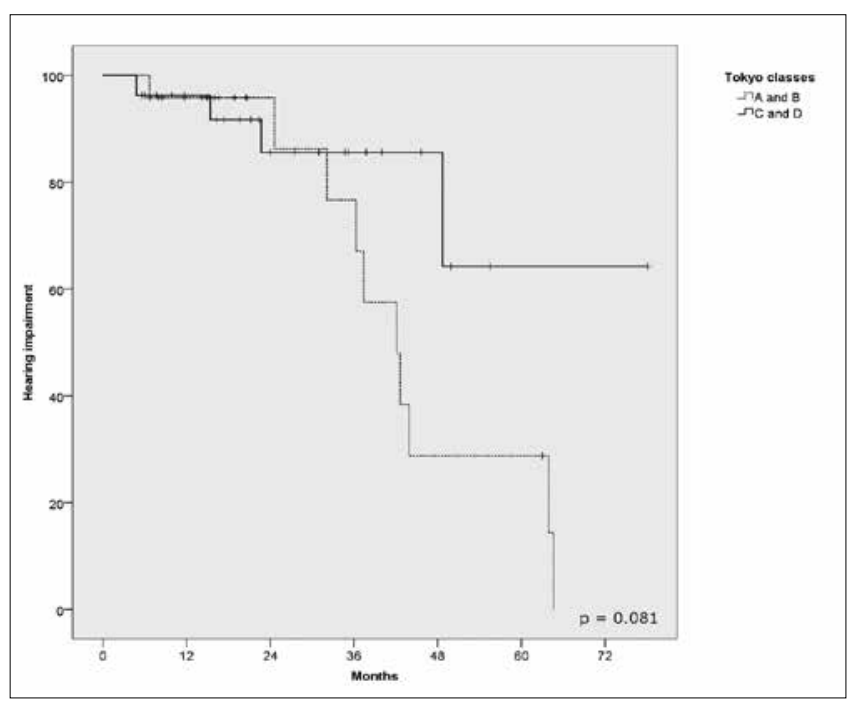

Fig. 5. Hearing impairment in relation to preoperative Tokyo class A-B or C-D hearing for the wait-and-see group of patients.

canal approach to the vestibular labyrinth. Post-operative major complications occurred in two cases $(5.4 \%)$, one epidural haematoma, which required revision of the extradural surgical field and one transient cerebellar oedema, which resolved after medical therapy and an early external drain that was removed after 3 days. No neurologic sequalae or other complications were observed. Cerebrospinal fluid leak was observed in two cases (5.4\%), which resolved spontaneously in one case and required surgical revision in the other. Postoperative facial nerve function was grade II HB after HPS in one patient with a 10 $\mathrm{mm}$ extrameatal tumour, who recovered to grade I HB 4 months after surgery; and grade III HB in the patient with preoperative facial nerve weakness. No recurrences were observed at last follow-up.

\subsubsection{Translabyrinthine surgery}

Seventeen patients -10 male and 7 female - with a mean age of $52.5 \pm 14.0$ years underwent translabyrinthine surgery. Eleven $(64.7 \%)$ patients came from the wait-and-see group, with a median time from diagnosis to treatment of 38.5 months (IQR 21-54 months). The other 6 patients were directly referred for surgery due to intractable vertigo (4 cases), planned CI (1 case), or facial nerve palsy ( 1 case). Patients arriving from the observation group had a significantly lower median PTA (55 dB, IQR 39-58 dB) than those referred for primary translabyrinthine surgery (75 dB, IQR 62-87.5 dB) $(\mathrm{p}=0.005)$.

\subsubsection{Hearing preservation surgery (HPS)}

The mean age in the group of 19 patients who underwent HPS was $47.5 \pm 10.4$ years. All candidates for HPS belonged to Tokyo and AAO-HNS hearing class A or B. The median PTA at diagnosis was $21.5 \mathrm{~dB}$, (IQR 12.5- 
Table V. Patients' characteristics in the HPS group, stratified by compliance with protocol.

\begin{tabular}{|c|c|c|c|}
\hline & $\begin{array}{c}\text { In-protocol }(n=13) \\
n(\%)\end{array}$ & $\begin{array}{c}\text { Off-protocol }(n=6) \\
n(\%)\end{array}$ & $p$ value \\
\hline Male & $9(69)$ & $4(67)$ & 1.00 \\
\hline Age at diagnosis (years), mean \pm SD & $44.7 \pm 8.3$ & $52.2 \pm 13.0$ & 0.145 \\
\hline $\begin{array}{l}\text { Median time from diagnosis } \\
\text { to treatment (months) }\end{array}$ & 6.3 & 5.8 & 0.357 \\
\hline Extrameatal tumour & $6(46)$ & $6(100)$ & $0.044^{*}$ \\
\hline CPA tumour size $(\mathrm{mm})$, mean $\pm \mathrm{SD}$ & $7.2 \pm 2.9$ & $8.7 \pm 3.3$ & 0.375 \\
\hline Baseline PTA (dB), median & 15.0 & 33.2 & 0.244 \\
\hline Postoperative PTA (dB), median & 36.2 & 71.8 & $0.022^{*}$ \\
\hline Maintained Tokyo hearing class A-B & $10(77)$ & $2(33)$ & 0.129 \\
\hline Maintained AAO-HNS hearing class A-B & $11(85)$ & $2(33)$ & $0.046^{*}$ \\
\hline
\end{tabular}

*Statistical significance

$32.5 \mathrm{~dB}$ ). Concerning postoperative hearing outcomes, Tokyo class A-B was maintained in 12 of the 19 patients (63\%), and AAO-HNS class A-B was maintained in 13 patients (68\%); 2 patients became Tokyo class D postoperatively, and were fitted with hearing aids; the remaining 5 cases became deaf.

Our preoperative inclusion criteria for HPS were met for 13 in-protocol cases $(68.4 \%)$. When hearing outcome was assessed by in- and off-protocol group (Table V), the success rate was $77 \%$ for the in-protocol patients, and 33\% for the off-protocol group according to the Tokyo classification. When the AAO-HNS hearing classification system was considered, the success rate for in-protocol patients was $85 \%$, differing significantly from the HPS results success rate $(33 \%)$ for the off-protocol group $(p=0.046)$. A significant difference in postoperative median PTA between the two groups was also observed $(p=0.022)$.

\section{Discussion}

This report focuses on the indications for the currentlyavailable treatment approaches to small $\mathrm{AN}$, and the results achieved in our series. The outcomes can only be interpreted in the light of the protocol adopted, which gives priority to the hearing function as the only discriminating factor among the various treatment options. Our management of small AN is discussed within this frame.

In the present series, 61 patients were assigned to the waitand-scan policy, 25 were referred directly for translabyrinthine surgery or HPS. The wait-and-see group showed an overall tumour growth rate of $28 \%$ over a median followup of 25 months. The patients under observation had small tumours and poor hearing, or were patients who had previously refused or were poor candidates for surgery. When tumour growth warranted active therapy, the indication was for HPS if still feasible, or a translabyrinthine approach or
RT otherwise. Hearing deteriorated in $28 \%$ of cases in the wait-and-see group, regardless of tumour growth. Tokyo classes A and B were maintained in 58\% of cases (14 of 24 patients), while classes $\mathrm{C}$ and $\mathrm{D}$ were maintained in $85 \%$ of cases. These results suggest that adopting a wait and see policy at diagnosis could be more appropriate for patients whose hearing is already impaired, while patients with a good hearing function, likely to worsen over time, could benefit more from active hearing preservation policies.

Our current overall success rate with HPS (postoperative class A-B hearing) was $63 \%$ according to the Tokyo classification and $65 \%$ to the AAO-HNS hearing grading system. In the literature, the results of hearing preservation surgery differ widely. Overall rates of success are reported after both the retro-sigmoid and middle cranial fossa approaches within a range of $46 \%$ and $82 \%$, regardless tumour size ${ }^{10}$. Considering only small AN $(\leq 15 \mathrm{~mm}$ in the CPA) and according to the AAO-HNS classification, preoperative classes A and B were maintained in $46 \%$ to $85 \%$ of cases ${ }^{13-16}$.

In our series, hearing function was preserved in $77 \%$ of cases within the preoperative limits of $30 \mathrm{~dB}$ PTA / 70\% SDS - normal or slightly altered ABR - size up to $10 \mathrm{~mm}$ in the CPA (in-protocol). In the off-protocol group the success rate dropped to $33 \%$. These results are similar to those of previous experiences ${ }^{10-12}{ }^{17}$. In-protocol patients achieving a class A-B outcome after HPS experienced hearing deterioration in $23 \%$ of cases according to the Tokyo classification, and $15 \%$ according to the AAO-HNS. This rate appears to be worse than observation and RT in the short term ${ }^{12}{ }^{17}$. The long-term hearing outcome is not yet available for the present sample, but in a previous series of 200 cases ${ }^{10}$ with a 6- to 21 -year follow-up (mean 14, median 9 years) it was as follows: postoperative AAO-HNS class A cases maintained class A or B in $92 \%$ of cases, and deteriorated to class C or D in $8 \% ; 87 \%$ 
of class A and B cases remained A or B, while $13 \%$ deteriorated to class $\mathrm{C}$ or D. Similarly, Wang and colleagues reported an $84 \%$ rate of preservation for postoperative AAO-HNS classes A and B at 5-year follow-up ${ }^{15}$. These are the data to compare with the rates of long-term loss of classes A and B reported by Kirchmann (66\%), and Stangerup $(56 \%)^{24}$.

In principle, it was the natural history of AN that dictated our treatment approach: most small tumours do not grow, but hearing function declines inexorably regardless of tumour growth. The wait-and-see policy is justified by the finding of no tumour growth for a considerable proportion of small tumours ${ }^{124518}$ despite progression of hearing loss. Followup seems to be the most predictable variable when assessing hearing outcome - whatever the therapeutic approach - because hearing decline is inescapable when tumours are observed over a long period of time.

Results obtained with RT are likewise promising in the short term ${ }^{51920}$, but diminish with time ${ }^{3}$, as shown by the $23 \%$ of cases with class A and B hearing preservation (serviceable hearing, 50/50\%) in the long term. RT remains an option in cases when patients prefer it or if surgery carries a high risk. The short-term results of microsurgery are worse than with observation or RT, but they merge with the more conservative treatments in the longer term, and it is selfevident that they should be considered more durable ${ }^{5}$. The variability of the results obtained in the surgical series is the main argument supporting the claim that RT is generally superior in terms of preserving hearing function ${ }^{19} 20$. HPS success rates also depend on the surgical team and are strongly influenced by preoperative patient selection ${ }^{514}{ }^{17}$. The comparison of unsuccessful HPS outcomes with that of RT series can be "misleading" ${ }^{20}$, since the heterogeneity of results is higher in the surgical group and if only the worse surgical series are considered for comparison, any conclusive statement is biased. Moreover, the effectiveness of RT should be determined in terms of disease control in the long-term, and only confined to tumours with documented growth ${ }^{21}$.

Proponents of each therapeutic approach may have their own way of assessing patients and comparing results in the attempt to investigate success and failure rates. It is nonetheless generally agreed that the outcomes in terms of survival, neurological losses and facial nerve preservation are good and much the same whatever the therapy. The difference lies in hearing preservation.

We tried to compare the results obtained with our multioption strategy as opposed to published data on the observation alone strategy. The most recent paper from a Danish group ${ }^{4}$ on the natural history of intrameatal AN reported long-term data on tumour growth and hearing. Despite a high rate of patient loss to follow-up (more than 50\%), this study is one of the most relevant long-term reports on the observation strategy for intrameatal AN. Intrameatal AN growth was reported in $37 \%$ of cases, extrameatal growth in $23 \%$ and the need to switch to active therapy was $15 \%$. AAO-HNS class A hearing ( $30 \mathrm{~dB} / 70 \%$ SDS) was maintained in $47 \%$ and $17 \%$ of cases at 5- and 10-year followup, respectively, and serviceable hearing ( $50 \mathrm{~dB} / 50 \% \mathrm{SDS})$ in $47 \%$ and $34 \%$, respectively. Hearing was class $\mathrm{C}$ in $66 \%$ of cases. The group of cases under observation with $100 \%$ SDS and a mean PTA of $46 \mathrm{~dB}$ at diagnosis, maintained $70-100 \%$ SDS at 10 years in $77 \%$ of cases, but no details were provided on the intensity at which the score was obtained. or the worsening PTA ${ }^{4}$. It is generally agreed that combining PTA and SDS enables a better, more complete judgement of the quality of functional hearing.

The main weaknesses of the present study are considered. The article reports on a monocentric series of small AN with a small number of patients and limited followup time, too short to draw any definitive conclusion, but enough to define a trend that needs further confirmation over time.

Moreover, the results were at least partially influenced by the specific institutional practices, therefore reducing their generalisability and introducing a bias in the treatment option, even if objective pre-treatment conditioning parameters were defined. A selection bias occurs in the present series as in every planned prospective study where randomisation of treatment is not feasible.

A multicentre collaboration among surgical experienced centres is advocated in order to achieve more robust results.

\section{Conclusions}

The various treatment options available for small AN provide good results in terms of disease control and complications, while their benefits on hearing outcome are still debated. A multi-option strategy combining both observation and active treatment (HPS or traditional surgery), according to a pre-treatment selection of patients, as related to our institutional hearing-focused protocol, appeared to provide better results than a single-modality option alone. In patients diagnosed early with small $\mathrm{AN}$, proactive treatment can either preserve the hearing function through HPS or rehabilitate it with a translabyrinthine surgery and hearing aids. Patients with postoperative hearing worse than class A or B might benefit from hearing rehabilitation with hearing aids, so whether a preserved class $\mathrm{C}$ aided hearing can be considered success or failure is debatable. HPS is advisable with an expected good outcome when preoperative hearing and tumour size are within the ranges of 
$\mathrm{PTA} \leq 30 \mathrm{~dB}, \mathrm{SDS} \geq 70 \%$ and $\leq 10 \mathrm{~mm}$ in the CPA. Otherwise, observation seems to be the choice, as shown by the poor results of pre-treatment unfavourable cases. Under a wait-and-see policy, hearing remains adequately stable in the short term, but seems to become unsatisfactory over time. Long-term hearing results are advisable, as they appear to be the determining outcome measure in selecting treatment options.

\section{Acknowledgements}

The authors thank Dr. Frances Coburn for the English revision of the paper.

\section{References}

1 Stangerup SE, Caye-Thomasen $\mathrm{P}$, Tos M, et al. The natural history of vestibular schwannoma. Otol Neurotol 2006;27:547-52.

2 Stangerup SE, Tos M, Thomsen J, et al. Hearing outcomes of vestibular schwannoma patients managed with 'wait and scan': predictive value of hearing level at diagnosis. J Laryngol Otol 2010;124:490-4.

3 Carlson ML, Link MJ, Wanna GB, et al. Management of sporadic vestibular schwannoma. Otolaryngol Clin North Am 2015;48:407-22.

4 Kirchmann M, Karnov K, Hansen S, et al. Ten-year followup on tumor growth and hearing in patients observed with an intracanalicular vestibular schwannoma. Neurosurgery 2017;80:49-56.

5 Golfinos JG, Hill TC, Rokosh R, et al. A matched cohort comparison of clinical outcomes following microsurgical resection or stereotactic radiosurgery for patients with smalland medium-sized vestibular schwannomas. J Neurosurg 2016;125:1472-82.

6 Martini A, Marioni G, Zanoletti E, et al. YAP, TAZ and AREG expression in eighth cranial nerve schwannoma. Int J Biol Markers 2017;32:e319-e24.

7 Kanzaki J, Tos M, Sanna M, et al. New and modified reporting systems from the consensus meeting on systems for reporting results in vestibular schwannoma. Otol Neurotol 2003;24:642,8.

8 American Academy of Otolaryngology-Head and Neck Surgery Foundation, INC. Committee on Hearing and Equilibrium guidelines for the evaluation of hearing preservation in acoustic neuroma (vestibular schwannoma). Otolaryngol Head Neck Surg 1995;113:179-80.
9 House JW, Brackmann DE. Facial nerve grading system. Otolaryngol Head Neck Surg 1985;93:146-7.

10 Mazzoni A, Zanoletti E, Calabrese V. Hearing preservation surgery in acoustic neuroma: long-term results. Acta Otorhinolaryngol Ital 2012;32:98-102.

11 Mazzoni A, Calabrese V, Danesi G. A modified retrosigmoid approach for direct exposure of the fundus of the internal auditory canal for hearing preservation in acoustic neuroma surgery. Am J Otol 2000;21:98-109.

12 Mazzoni A, Zanoletti E, Denaro L, et al. Retrolabyrinthine meatotomy as part of retrosigmoid approach to expose the whole internal auditory canal: rationale, technique and outcome in hearing preservation surgery for vestibular schwannoma. Oper Neurosurg 2018;14:36-44.

13 Scheich M, Ehrmann-Müller D, Shehata-Dieler W, et al. Hörergebnisse nach transtemporaler Resektion kleiner (T1/ T2) Akustikusneurinome. HNO 2017;65:751-7.

14 Yamakami I, Ito S, Higuchi Y. Retrosigmoid removal of small acoustic neuroma: curative tumor removal with preservation of function. J Neurosurg 2014;121:554-63.

15 Wang AC, Chinn SB, Than KD, et al. Durability of hearing preservation after microsurgical treatment of vestibular schwannoma using the middle cranial fossa approach. $\mathrm{J}$ Neurosurg 2013;119:131-8.

${ }_{16}$ Gjuric M, Rudic M. What is the best tumor size to achieve optimal functional results in vestibular schwannoma surgery? Skull Base 2008;18:317-25.

17 Mazzoni A, Biroli F, Foresti C, et al. Hearing preservation surgery in acoustic neuroma. Slow progress and new strategies. Acta Otorhinolaryngol Ital 2011;31:76-84.

18 Patnaik U, Prasad SC, Tutar H, et al. The long-term outcomes of wait-and-scan and the role of radiotherapy in the management of vestibular schwannomas. Otol Neurotol 2015;36:638-46.

19 Carlson ML, Jacob JT, Pollock BE, et al. Long-term hearing outcomes following stereotactic radiosurgery for vestibular schwannoma: patterns of hearing loss and variables influencing audiometric decline. J Neurosurg 2013;118:579-87.

20 Myrseth E, Moller P, Pedersen PH, et al. Vestibular schwannoma: surgery or gamma knife radiosurgery? A prospective, nonrandomized study. Neurosurgery 2009;64:654,61.

21 Miller T, Lau T, Vasan R, et al. Reporting success rates in the treatment of vestibular schwannomas: are we accounting for the natural history? J Clin Neurosci 2014;21:914-8. 